ORIGINAL ARTICLE

\title{
The appearance of kissing contusion in the acutely injured knee in the athletes
}

\author{
I P Terzidis, A G Christodoulou, A L Ploumis, S R Metsovitis, M Koimtzis, P Givissis
}

Br J Sports Med 2004;38:592-596. doi: 10.1136/bjsm.2003.006718

See end of article for authors' affiliations ......................

Correspondence to: Dr loannis P Terzidis, 36 Ermou Street 54623, Thessaloniki,

Greece:jonterz@otenet.gr

Accepted 8 July 2003

\begin{abstract}
Background: Bone contusions are often identified at magnetic resonance imaging (MRI) in the acutely injured knee. Contusions of both surfaces of the joint are known as kissing contusions.

Objective: To determine the frequency, type, and distribution of kissing contusions occurring in association with injuries of the knee joint.

Methods: 255 MRI examinations in athletes with acutely injured knees (197 men; 58 women; mean age 24.2 years) were reviewed by two independent examiners; $219 \mathrm{MRIs}$ were done within the first month after the injury and 36 within two to four months. None of the knees had been injured before. No fractures were present on $x$ ray.

Results: Bone contusions were diagnosed in 71 cases (27.8\%); 55 (22.5\%) were identified as single contusions and $16(6.3 \%)$ as kissing contusions. Eight of the kissing contusions were associated with anterior cruciate ligament tears, three with menisceal tears, four were isolated lesions, and one was delayed, following a menisceal tear. The 32 bone contusions (16 kissing contusions) were located as follows: lateral femoral condyle ( $n=14 ; 8$ type I, 6 type II); lateral tibial condyle $(\mathrm{n}=9 ; 3$ type I, 1 type II, 5 type III); medial tibial condyle ( $n=7 ; 2$ type I, 5 type III); medial femoral condyle $(n=2 ;$ both type I). The associated injuries were confirmed by arthroscopy in 12/16 patients.

Conclusions: Kissing contusion is a significant injury often associated with ligamentous or menisceal injuries. Type I lesions are most common on the lateral femoral condyle and type III on the lateral tibial condyle.
\end{abstract}

B one contusion is a finding substantiated by magnetic resonance imaging (MRI) studies. The entity is recognised as a focal signal abnormality in the subchondral bone marrow, and the appearances are thought to represent microtrabecular fractures, haemorrhage, and oedema of the marrow, without disruption of the adjacent cortices or overlying articular cartilage (fig 1A). Unlike a fracture, bone contusion rarely involves both cancellous and compact cortical bone. These osseous injuries may result from a direct blow to the bone, from compression forces of adjacent bones impacting one another, or from traction forces that occur during an avulsion injury.

The terms "bone contusion," "bone bruise," and "bone marrow oedema" have appeared in published reports for at least a decade and seem to be used synonymously. MRI sensitivity for bone contusion is reported to be $91-96 \%$ and specificity $86-96 \%{ }^{1}$

With respect to the frequency of bone contusion, Binfield and Williams ${ }^{2}$ reported a rate of $4 \%$ in the immature knee, while Bretlau et $a l^{3}$ reported a rate of $65 \%$ in the acutely injured knee, and Perrin $e t ~ a l^{4}$ a rate of $58 \%$ associated with more serious injuries (menisceal tears, ligamentous tears). Bone contusions in both surfaces of the knee (kissing contusion) are rather rare complex injuries of the knee, and their importance has not been well documented. These characteristic lesions, which occur on both the femoral and tibial condyle, are of great importance not only in that they are often a sign of more serious associated injury, but also because they may give rise to prolonged symptoms.

Our aim in this study was to determine the frequency of kissing contusions in acutely injured knees in young athletes, and the type and distribution of these lesions in the overall spectrum of bone contusions following intra-articular and extra-articular injuries.

\section{METHODS}

From April 1996 to December 2000, 255 MRI examinations were undertaken in addition to clinical examination in 255 athletes with acute knee injuries (197 men and 58 women), mean age of 24.2 years (range 16 to 32 ). These athletes were competing in various different sports (table 1). Of the 255 MRI investigations, 219 were done within the first month after the acute knee injury and 36 within two to four months. None of the patients had previous knee injuries and no fracture was present on $x$ ray examination.

The MRI examinations were done using a Magnetom Impact VB33D (Siemens, Ehrlangen, Germany) 1.0 Tesla, using T1-W SE and T2-W FSE sequences. According to Mink and Deutsch, ${ }^{5}$ bone contusions are trauma induced, geographical, non-linear, and poorly defined areas of decreased signal intensity on $\mathrm{Tl}$ weighted imaging, increasing irregularly on T2 weighted sequences. A short while after that report, the same investigators referred to a new technique used in the assessment of bone marrow disorders. ${ }^{6}$ With this method, called short TI inversion recovery (STIR), an inversion time interval is chosen in order to suppress (null) the signal from medullary fat; the areas of abnormal bone marrow show increased signal intensity and are contrasted with the low signal intensity of suppressed marrow fat. Currently, we use fat suppressed T2 or T2 FSE IR sequences to improve the detection of bone marrow abnormalities, ${ }^{78}$ because of their comparable accuracy and short imaging time characteristics which make these techniques superior to conventional STIR sequences.

We classified the types of bone contusion according to Lynch et al. ${ }^{9}$ In type I lesions there is a loss of signal intensity on $\mathrm{Tl} \mathrm{W}$ or proton density sequences and an increased signal on T2 W, more evident in fat suppressed T2 or T2 FSE IR images; the area showing such variations in signal intensity 
is located primarily within the medullary cavity of the bone, without cortical interruption. Type II and type III lesions show the same signal intensity characteristics as type I, but type II is associated with interruption of the black cortical line, and type III is strictly located in the region of bone immediately adjacent to the cortex without any definite cortical interruption.

A menisceal tear was diagnosed on proton density images or, best, on T2 weighted sequences, when the images typically showed a disruption of the triangular low intensity signal as an irregular high intensity signal (vertical or horizontal)
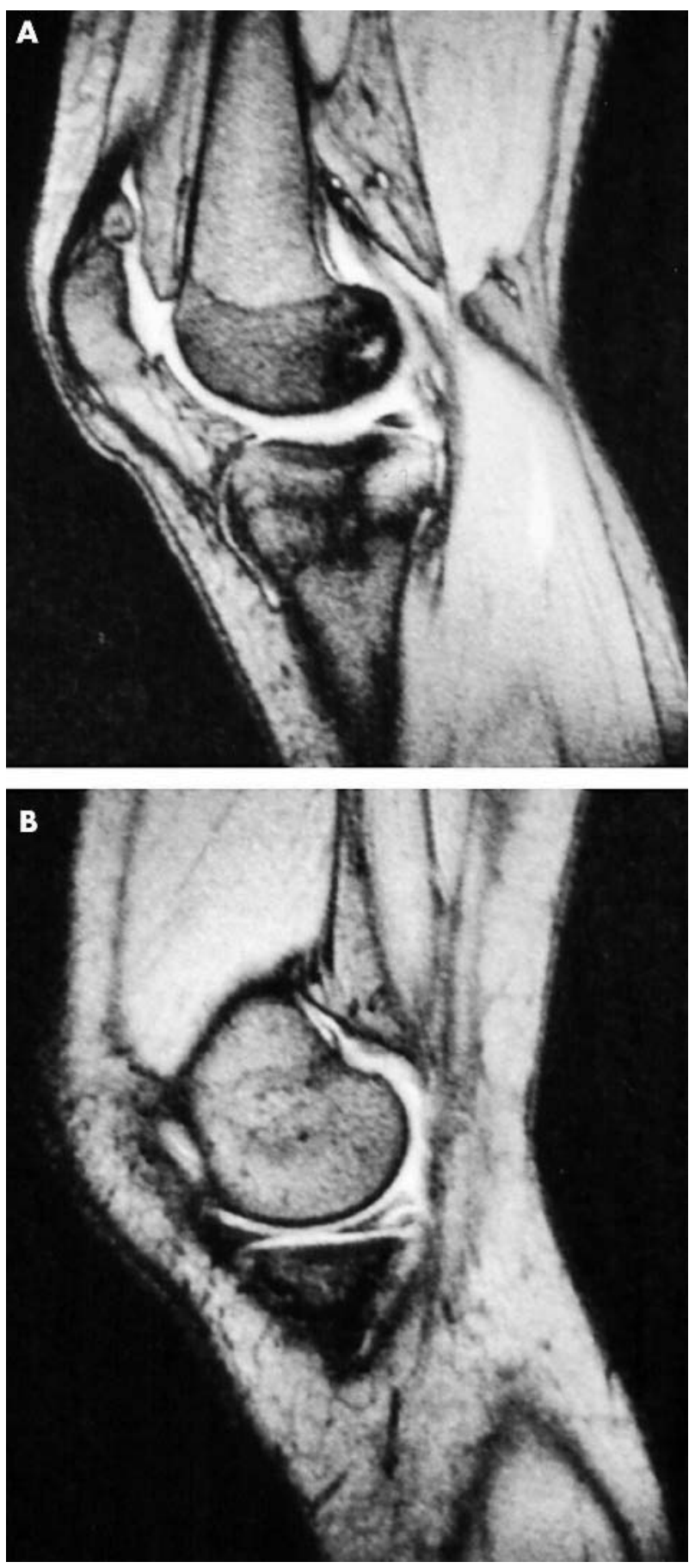

Figure 1 Sagittal proton density images showing (A) typical kissing contusion with bone bruise areas of low density signal in the medial femoral condyle and the posterior lip of the medial tibial plateau. (B) Note the menisceal tear.
Table 1 Knee injuries and athletic activities in 255 patients

\begin{tabular}{llll}
\hline & Male & Female & Total \\
\hline Football & 103 & 14 & 117 \\
Basketball & 35 & 11 & 46 \\
Volleyball & 1 & 2 & 3 \\
Handball & 12 & 4 & 16 \\
Runners & 13 & 8 & 21 \\
Judo/Tae kwo do & 9 & 4 & 13 \\
Rowing & 8 & 1 & 9 \\
Skiing & 11 & 14 & 25 \\
Weight lifting & 3 & - & 3 \\
Sailing & 2 & - & 2 \\
Total & 197 & $\mathbf{5 8}$ & $\mathbf{2 5 5}$ \\
\hline & & &
\end{tabular}

extending to the margin of the surface of the meniscus (fig 1B). On the other hand if the images showed an increased signal intensity only in the body (central area) of the meniscus without extension, this could be the result of a mixoid or degenerative lesion.

A cruciate ligament injury was diagnosed when an intermediate signal on $\mathrm{Tl}$ weighted imaging and an increase in signal on $\mathrm{T} 2$ weighted imaging was present. Complete rupture was diagnosed when there was absence of any intact fibres along the normal course of the ligament, and a partial rupture if some continuous fibres were present.

Collateral ligament injuries were diagnosed when there was increased signal intensity in the ligament area on coronal proton density and T2 weighted spin echo images. Axial T2 weighted fast spin echo may be used to complement the coronal images.

All the MRI sequences were analysed by two independent examiners-one senior radiologist and the head of our orthopaedic surgery team. Interobserver agreement was expressed by the $\kappa$ statistic (two level $\kappa$ ), with MRI interpreted as positive or negative for bone contusion, and for increased signal intensity for menisceal and ligamentous lesions. According to Davies et al, ${ }^{10}$ interobserver agreement is perfect when $\kappa=>0.80$; good when $\kappa=0.80-0.61$; moderate when $\kappa=0.60-0.41$; fair when $\kappa=0.40-0.21$; and poor when $\kappa=<0.21$.

Arthroscopy was done in 12 of the 16 patients who suffered from kissing bone contusion, and the intra-articular lesions that were diagnosed on MRI were confirmed. Patients were followed up with clinical examinations for a mean of 31 months (range 24 to 36 )

\section{RESULTS}

Bone contusions were diagnosed in 71 cases $(27.8 \%)$ in a total of 225 MRI investigations undertaken on the same number of injured knees. Interobserver agreement was perfect ( $\kappa=0.921,95 \%$ confidence interval, 0.817 to 1.0 ).

\section{Kissing contusions}

In 16 of the 225 cases (6.3\%), bone contusions were found on both femoral and tibial surfaces (kissing contusions) (fig 2), nine in the lateral compartment (fig 3, panels A and B), two in the medial compartment, and five in the lateral femoral condyle associated with the medial tibial condyle (table 2). Interobserver agreement was perfect $(\kappa=0.882 \quad(0.792$ to $1.0))$.

\section{Ligamentous injuries}

In eight of the 16 cases of kissing contusion, there was an associated complete rupture of the anterior cruciate ligament. In seven of these cases, this injury was combined with a lesion of the medial collateral ligament and in one case with a 


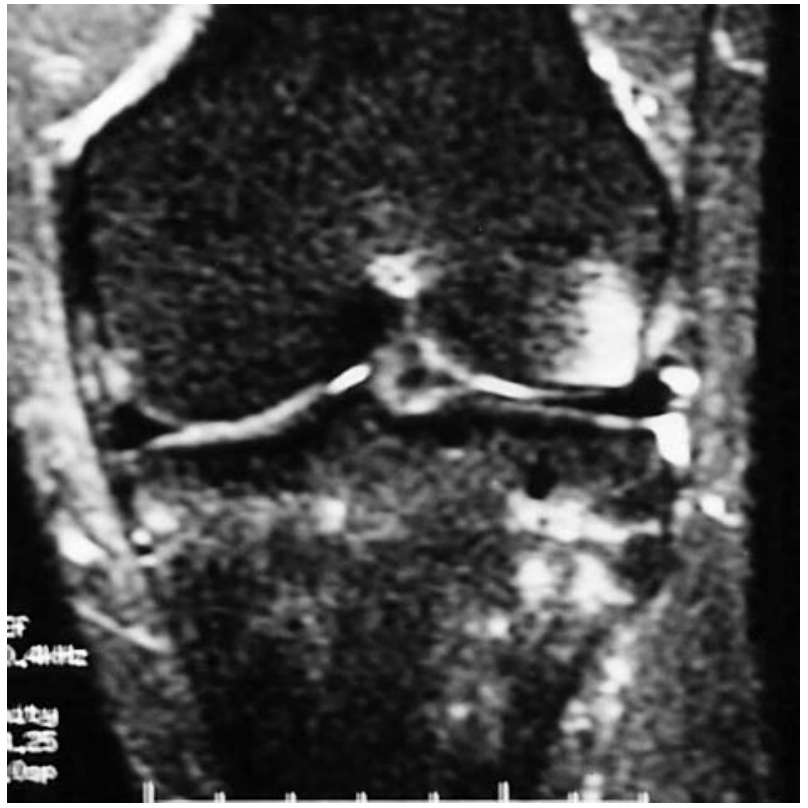

Figure 2 Coronal FSE inversion recovery image, showing kissing contusion in the lateral compartment as areas of high intensity signal. The surrounding medullary bone (fatty marrow) is suppressed and appears as low density (null) signal.

lesion of the lateral collateral ligament. In four of the cases (table 2, cases 4, 5, 12, and 13), the bone contusion was located on the terminal sulcus on the lateral femoral condyle (type I and type II) and on the posterolateral tibial condyle (type III). In three cases (table 2, cases 14, 15, and 16), the bone contusion was located on the lateral femoral condyle (type I and type II) and on the posterior lip of the medial tibial condyle (type III). One case (table 2, case 6) involved the medial compartment, with a type I lesion on the medial femoral condyle and on the posterior lip of the medial tibial condyle.

\section{Isolated lesions}

In four of the 16 cases (25\%), the kissing contusion was found as an isolated lesion. In three (table 2, cases 8,10 , and $11)$ the kissing contusion appeared in the lateral compartment, with a type II lesion on the femoral condyle and a type I lesion on the tibial condyle. In one case (table 2, case 9), a type I lesion was found on the lateral femoral condyle and a type III lesion on the medial tibial condyle.

\section{Menisceal lesions}

In three of the 16 cases (18.7\%) (two in the lateral and one in the medial compartment), bone contusion was found to be associated with menisceal lesions. Two cases (table 2, cases 1 and 2) were related to unilateral menisceal lesions (one oblique in the medial meniscus and one radial tear in the lateral meniscus), and one case (table 2, case 3 ) was related to bilateral menisceal lesions (oblique in the lateral meniscus and bucket handle in the medial meniscus).

All contusions on the femoral condyle were type I; on the tibial condyle two contusions were type III and one was type II.

\section{Delayed appearance}

In one case (table 2, case 7 ), with bone contusion on the lateral femoral condyle (type I) associated with a tear of the medial meniscus and partial rupture of the medial collateral ligament, bone contusion (type I) was found on the medial tibial condyle on a new MRI after four months. The initial
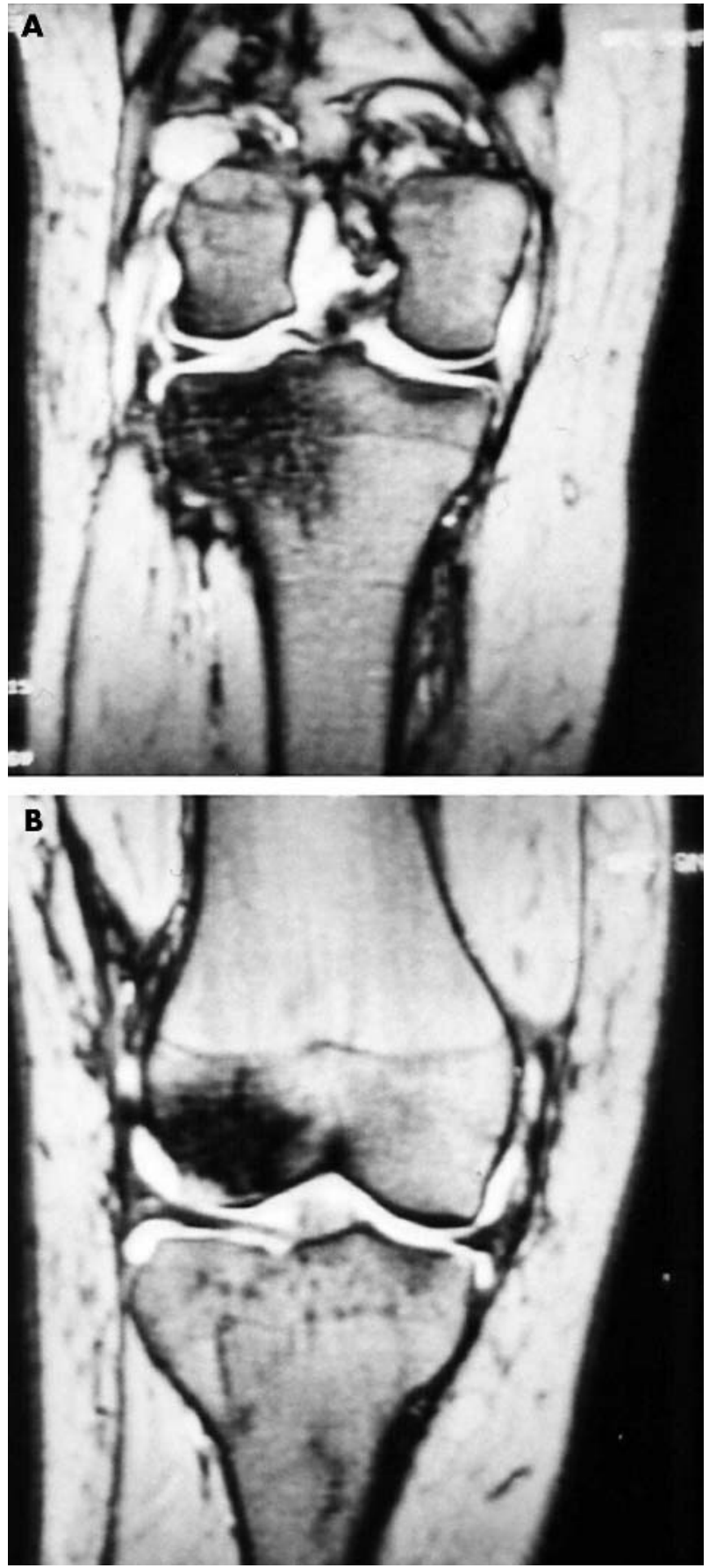

Figure 3 Coronal proton density images showing the kissing contusion low density signal areas (arrows) on the lateral femoral condyle (A). A more posterior section (B) shows the contusion on the lateral tibial condyle.

bone contusion on the lateral femoral condyle was still detectable but was more diffuse than on the initial MRI.

\section{Operative procedure}

The ACL ruptures were confirmed arthroscopically in all cases, and reconstitution was done using BPTB or hamstring tendon autografts. Partial meniscectomy was undertaken in all cases with menisceal lesions. Once the associated injuries were treated arthroscopically, the remaining clinical symptoms of bone contusion settled within three to seven months in cases with menisceal tears, in four to nine months in cases 
Table 2 Location of kissing contusion in combination with intra-articular or extraarticular injuries

\begin{tabular}{|c|c|c|c|c|c|c|c|c|c|}
\hline Case & LFC & MFC & LTC & MTC & $\mathrm{ACL}$ & LM & MM & $\mathrm{MCL}$ & LCL \\
\hline 1 & 1 & & III & & & Radial & & & \\
\hline 2 & I & & $\|$ & & & & Oblique & & \\
\hline 3 & & 1 & & III & & Oblique & Buck-h & & \\
\hline 4 & 1 & & III & & Complete & & & Complete & \\
\hline 5 & i & & III & & Complete & & & Partial & \\
\hline 6 & & I & & I & Complete & & & & Partial \\
\hline 7 & 1 & & & I & & & Horizontal & Partial & \\
\hline 8 & $\|$ & & I & & & & & & \\
\hline 9 & I & & & III & & & & & \\
\hline 10 & $\|$ & & I & & & & & & \\
\hline 11 & $\|$ & & i & & & & & & \\
\hline 12 & $\|$ & & III & & Complete & & & Complete & \\
\hline 13 & 1 & & III & & Complete & & & Partial & \\
\hline 14 & ॥ & & & III & Complete & & & Partial & \\
\hline 15 & ॥ & & & III & Complete & & & Partial & \\
\hline 16 & I & & & III & Complete & & & Complete & \\
\hline
\end{tabular}

with anterior cruciate ligament tears, and in three to six months in isolated cases, with an overall mean of 5.2 months of recovery time (range 3 to 9 ).

\section{Single contusions}

In the 55 cases with a single bone contusion (table 3), 11 $(20 \%)$ were present as isolated injuries, 17 (30.9\%) were associated with complete anterior cruciate ligament rupture, five $(9 \%)$ with partial anterior cruciate ligament rupture, eight $(14.5 \%)$ with a medial collateral ligament tear, eight $(14.5 \%)$ with menisceal tears, four $(7.3 \%)$ with medial collateral and anterior cruciate ligament rupture, and two $(3.6 \%)$ with a lateral collateral ligament tear. Forty of the 55 lesions $(72.7 \%)$ were found on the lateral femoral condyle, 11 $(20 \%)$ on the medial femoral condyle, three $(5.4 \%)$ on the lateral tibial condyle, and one $(1.9 \%)$ on the medial tibial condyle.

The interobserver agreement was perfect $(\kappa=0.912(0.821$ to 1.0$)$ ).

\section{DISCUSSION}

There have been only a few reports describing pathological and radiological correlations in patients with bone marrow abnormalities on MRI. Bone contusions-which are considered to represent microtrabecular fractures, haemorrhage, and oedema of the subcortical bone marrow-are increasingly recognised through MR imaging of the acutely injured knee. Rangger et al described microfractures of cancellous bone, and oedema and bleeding in the fatty marrow. ${ }^{11}$ Kyung et $a l^{12}$ in their study of MRI characteristics and their histological correlations of bone injuries in young pigs, found that MR imaging could show changes in the bone marrow resulting from direct injury to the bone, and was a useful tool for evaluating the evolution of bone contusion.

The incidence of bone contusion detectable by MRI ranges from $14 \%^{2}$ up to $80 \%^{13}$ in patients with knee injuries. In simple injuries without menisceal or ligamentous rupture, the frequency of bone contusion appears to range between $4 \%{ }^{14}$ and $17 \% .{ }^{15}$ In the present series the frequency of single bone contusion was $22.5 \%$ and for the kissing contusion, $6.3 \%$-an overall frequency of $27.8 \%$.

Niall et al reported that the strongest correlation occurred between isolated anterior cruciate ligament injuries and lateral femoral condyle and posterolateral tibial condyle bone contusion (73\%). ${ }^{16}$ The most common bone contusion with all anterior cruciate injuries (isolated or complex) was on the lateral tibial condyle (82\%). According to Zeis et al, ${ }^{17}$ bone bruising was found in $72 \%$ of complete anterior cruciate ligament tears but in only $12 \%$ of partial tears.

In this series, $38 \%$ of single bone contusions were associated with complete anterior cruciate ligament tears (21/55: 17 with complete anterior cruciate ligament rupture; four with anterior cruciate plus medial cruciate rupture), and $9 \%$ with partial anterior cruciate ligament tears. However, $50 \%$ of the cases of kissing contusion were associated with anterior cruciate ligament ruptures, which explains the fact that kissing contusions are significant knee injuries.

The association of bone contusion with menisceal lesions is reported to be around $8 \% .{ }^{18}$ In the present series it was $14 \%$, and $31.2 \%$ in association with kissing contusions (five of the 16 cases: three tears in medial meniscus and two in the lateral), the medial meniscus being involved in the majority of cases.

Miller et al reported a bone contusion frequency of $45 \%$ in association with medial collateral ligament tears, ${ }^{19}$ while for a single contusion in the present series the figure was $20 \%$ (table 3) and for kissing contusions, 50\% (eight of 16: seven in acutely injured knees and one delayed). Kaplan et al proposed that contusion of the posterior lip of the medial tibial plateau and the occasionally associated contusion of the medial femoral condyle most probably occurs as a contrecoup injury, as the tibia reduces following an anterior cruciate

Table 3 Location of single bone contusion in combination with other soft tissue injuries

\begin{tabular}{|c|c|c|c|c|c|c|c|}
\hline & ACL complete & $\mathrm{ACL}$ partial & Lateral meniscus & Medial meniscus & MCL & LCL & Isolated \\
\hline Lateral femoral condyle & $18(32.7 \%)$ & $4(7.2 \%)$ & $1(1.8 \%)$ & $3(5.5 \%)$ & $11(20 \%)$ & $1(1.8 \%)$ & $6(10.8 \%)$ \\
\hline Medial femoral condyle & $3(5.5 \%)$ & $1(1.8 \%)$ & - & $4(7.2 \%)$ & - & $1(1.8 \%)$ & $2(3.6 \%)$ \\
\hline Lateral tibial condyle & - & - & - & - & $1(1.8 \%)$ & - & $2(3.6 \%)$ \\
\hline Medial tibial condyle & - & - & - & - & - & - & $1(1.8 \%)$ \\
\hline
\end{tabular}




\section{Take home message}

- Bone contusion of the knee is substantiated by MRI studies. These injuries may result from a direct blow to the bone or from compressive forces of adjacent bones impacting on one another during an avulsion injury. Bone contusions are commonly seen in patients with anterior cruciate ligament injuries (65\%) but they may also occur on their own or in association with other acute knee injuries.

- "Kissing contusion" refers to a bone contusion on both side of the knee joint. It is a rare (6.3\% in this series) but significant injury, often associated with ligamentous or menisceal tears. Disabling symptoms are often prolonged.

ligament rupture. ${ }^{20}$ In our study only one case of this type of injury was observed.

Most of the kissing contusions occur in the lateral joint compartment, involving the weight bearing surface of the lateral femoral condyle and the posterior aspect of the lateral tibial plateau, and they are mainly seen in association with anterior cruciate ligament and medial collateral ligament tears. ${ }^{20}$ Bone contusions in the medial compartment of the knee are far less common and have not been well described in published reports. In our series, two of the 16 cases of kissing contusion were in the medial compartment.

There is little published information about the type of bone bruising and whether the injuries are intra-articular or extraarticular in kissing contusion. In our study, 32 bone contusions (16 kissing contusions) were located as follows: $14(43.7 \%)$ on the lateral femoral condyle (eight type I, six type II); nine $(28.1 \%)$ on the lateral tibial condyle (three type I, one type II, five type III); seven (21.9\%) on the medial tibial condyle (two type I, five type III); and two (6.3\%) on the medial femoral condyle (both type I). It appears that type I lesions are most common on the femoral condyle (62.5\%) and type III on the tibial condyle $(62.5 \%)$.

In our series we found that menisceal lesions were associated with type I lesions on the femoral condyle and type II and III lesions on the tibial condyle, except for one case with a type I lesion on the medial femoral condyle and a type III on the medial tibial condyle.

In cases of anterior cruciate ligament tear, we found type I and II lesions on the femoral condyle and type III lesions on the tibial condyle (except for one case with type I). We found only type I lesions on the femoral condyle and types I, II, and III lesions on the tibial condyle in cases with menisceal tears. In most cases of isolated bone contusion, however, type II lesions were found on the femoral condyle and type I lesions on the tibial condyle. This could be explained by the mechanism of injury (pivot shift, hyperextension) or the varus/valgus position of the knee and the other associated lesions that occur at the time of injury.

Although the associated injuries in our patients were treated arthroscopically, the persistence of symptoms for a mean of 5.2 months (range 3 to 9) in bone contusion injuries indicates the need for prolonged rest.

\section{Conclusions}

Bone contusion of the knee is substantiated by MRI studies. It may give rise to confusion in the differential diagnosis of knee injuries. Kissing contusions are rare (16/225 (6.3\%)) but significant injuries, often associated with ligamentous or menisceal tears. Single bone contusions are seen more often $(55 / 225(21.5 \%))$. Type I lesions seem to be most common on the lateral femoral condyle and type III on the lateral tibial condyle.

\section{Authors' affiliations}

I P Terzidis, A G Christodoulou, A L Ploumis, S R Metsovitis, M Koimtzis, P Givissis, Department of Orthopaedics, "Hippokration" General Hospital of Thessaloniki, Thessaloniki, Greece

\section{REFERENCES}

1 Arndt WF, Truax AL, Barnett FM, et al. MR diagnosis of bone contusions of the knee: comparison of coronal T2-weighted fast spin-echo with fat saturation and fast spin-echo STIR images with conventional STIR images. Am J Roentgenol 1996;166:119-24.

2 Binfield P, Williams LA. The clinical and radiological outcome of MRI detected bone bruises of the immature knee. Third Congress of the European Federation of National Associations of Orthopaedics and Traumatology 1997, No P0804 [abstract]. Proc J Bone J Surg 1997:79-B2.

3 Bretlau T, Tuxoe J, Larsen L, et al. Bone bruise in the acutely injured knee. Knee Surg Sports Traumatol Arthrosc 2002; 10:96-101.

4 Perrin B, Nardacchione R, Bonaga S, et al. Acute knee block. Assessment with magnetic resonance, correlated with arthroscopy. Radiol Med (Torino) 1997;93:40.

5 Mink JH, Deutsch AL. Occult cartilage and bone injuries of the knee: detection, classification, and assessment with MR imaging. Radiology 1989; 170:823-9.

6 Deutsch AL, Mink JH. Magnetic resonance imaging of musculoskeletal injuries. Radiol Clin North Am 1989;27:983-1002.

7 Snearly WN, Kaplan PA, Dussault RG. Lateral-compartment bone contusions in adolescents with intact anterior cruciate ligaments. Radiology 1996; 198:205-8

8 Irizarry JM, Recht MP. MR imaging of the knee ligaments and the postoperative knee. Radiol Clin North Am 1997;35:45-76.

9 Lynch TCP, Crues JV, Morgan FW, et al. Bone abnormalities of the knee: prevalence and significance at MR imaging. Radiology 1989;171:761-6.

10 Davies M, Fleiss JL. Measuring agreement for multinomial data. Biometrics 1982;38:1047-51.

11 Rangger C, Kathrein A, Freund MC, et al. Bone bruise of the knee: histology and cryosections in 5 cases. Acta Orthop Scand 1998;69:291-4.

12 Kyung NR, Wook J, Young TK, et al. Bone bruises: MR characteristics and histological correlation in the young pig. Clin Imaging 2000;24:371-80.

13 Adalbeth T, Roos $\mathrm{H}$, Lauren $M$, et al. Magnetic resonance imaging, scintigraphy and arthroscopic evaluation of traumatic hemarthrosis of the knee. Am J Sports Med 1997;25:231-7.

14 Tung GA, Davis $L M$, Wiggins ME, et al. Tears of the anterior cruciate ligament: primary and secondary signs at MR imaging. Radiology 1993; 188:661-7.

15 Duncan JB, Hunter $H$, Purnell $M$, et al. Injured stable knee with acute effusion: MRI evaluation. J South Orthop Assoc 1996;5:13-19.

16 Niall D, Davis N, King L, et al. Short term clinical and radiological outcome of bone bruising identified on magnetic resonance imaging of the acutely injured knee. European Federation of National Associations of Orthopaedics and Traumatology [abstract]. Proc J Bone Joint Surg 1997:79-B2.

17 Zeiss J, Paley K, Murray K, et al. Comparison of bone contusion seen by MRI in partial and complete tears of the anterior cruciate ligament. J Comput Assist Tomogr 19:773-6.

18 Rangger C, Klestil T, Kathreinn A, et al. Influence of magnetic resonance imaging on indications for arthroscopy of the knee. Clin Orthop 1996;330:133-42.

19 Miller MD, Osborne JR, Gordon WT, et al. The natural history of bone bruises. A prospective study of magnetic resonance imaging-detected trabecular microfractures in patients with isolated medial collateral ligament injuries. Am J Sports Med 1998;26:15-19.

20 Kaplan PA, Gehl RH, Dussault RG. Bone contusions of the posterior lip of the medial tibial plateau (contrecoup injury) and associated internal derangements of the knee at MR. Imaging Radiol 1999;211:747-53. 